\title{
Comparison of analogue and electronic stethoscopes for pulmonary auscultation by internal medicine residents
}

\author{
Eric R. Gottlieb, ${ }^{1}$ Jason M. Aliotta, ${ }^{2}$ Dominick Tammaro ${ }^{1}$
}

\begin{abstract}
'Department of Medicine, Warren Alpert Medical School of Brown University, Rhode Island Hospital, Providence, Rhode Island, USA

2Division of Pulmonary, Critical Care and Sleep Medicine, Department of Medicine, Warren Alpert Medical School of Brown University, Rhode Island Hospital, Providence, Rhode Island, USA
\end{abstract}

\section{Correspondence to} Dr Eric R. Gottlieb, Department of Medicine, Warren Alpert Medical School of Brown University, Rhode Island Hospital, Providence, RI 02903, USA; egottlieb@gmail.com

Received 8 August 2018 Revised 16 October 2018 Accepted 27 October 2018 Published Online First 24 November 2018
ABSTRACT

Background Electronic stethoscopes are becoming more common in clinical practice. They may improve the accuracy and efficiency of pulmonary auscultation, but the data to support their benefit are limited.

Objective To determine how auscultation with an electronic stethoscope may affect clinical decision making.

Methods An online module consisting of six fictional ambulatory cases was developed. Each case included a brief history and lung sounds recorded with an analogue and electronic stethoscope. Internal medicine resident participants were randomly selected to hear either the analogue or electronic lung sounds. Numbers of correct answers, time spent on each case and numbers of times the recordings were played were compared between the groups who heard each mode of auscultation, with a $p$ value of less than 0.05 indicating statistical significance. Results 61 internal medicine residents completed at least one case, and 41 residents completed all six cases. There were no significant differences in overall scores between participants who heard analogue and electronic lung sounds ( $3.14 \pm 0.10$ out of 6 correct for analogue, $3.20 \pm 0.10$ out of 6 for electronic, $p=0.74$ ). There were no significant differences in performance for any of the six cases $(p=0.78)$, time spent on the cases $(p=0.67)$ or numbers of times the recordings were played $(p=0.85)$. Conclusion When lung sounds were amplified with an electronic stethoscope, we did not detect an effect on performance, time spent on the cases or numbers of times participants listened to the recordings.

\section{INTRODUCTION}

The stethoscope has been part of the standard patient examination ever since it was developed by Rene-Theophyle-Hyacinthe Laennec in $1816 .{ }^{1}$ Although some clinicians question its utility in the age of advanced imaging, it remains a cornerstone of the diagnostic process. Abnormal findings influence immediate clinical decision-making and dictate the need for further testing. The stethoscope is especially important in non-hospital settings, such as by emergency medical services and in ambulatory clinics where imaging is not immediately available. The act of auscultation is also an important ritual in the doctor-patient relationship. ${ }^{2}$

The stethoscope is routinely used to examine multiple organ systems and has other physical examination uses as well, including as a makeshift reflex hammer, ${ }^{3}$ a hearing aid for communication with deaf patients ${ }^{4}$ and a malingering detector. ${ }^{5}$
However, it has arguably the greatest immediate impact in general internal medicine when used for pulmonary auscultation. A variety of pathological conditions can be detected and treated based largely on auscultation, including pneumonia, asthma, chronic obstructive pulmonary disease (COPD) and congestive heart failure (CHF), but interpretation is unfortunately unreliable. ${ }^{6}$ Poor auscultation can adversely affect patient outcomes and increase healthcare expenditures. ${ }^{7}$ Electronic stethoscopes, first developed in the 1940s, may increase reliability by allowing for amplification and increasingly sophisticated computer analysis. ${ }^{8}$

Several different electronic stethoscopes are commercially available, and each device uses one of several different mechanisms for filtering and amplification. ${ }^{9}$ The Eko Core device, approved by the US Food and Drug Association in 2015, attaches in-line to the tubing of a Littmann Cardiology-grade stethoscope. It amplifies sound transmitted from the chestpiece and projects it through a speaker attached to the other end of the tubing to be heard through binaurals like a traditional analogue stethoscope. ${ }^{10}$ It also has Bluetooth capability, which can send the recording to a smartphone app that saves and replays it with a phonogram.

The objective of this study was to determine whether electronic amplification with such a device would lead to more accurate and reliable interpretation of lung sounds and consequently result in better clinical decision making. This could influence clinicians' decisions as to whether to use electronic stethoscopes and indicate whether hospitals and residency programmes should consider supporting their implementation.

\section{METHODS \\ Study protocol}

Patients with a variety of lung pathology were identified from a medical intermediate care unit. Patients agreed to have their lung sounds anonymously recorded. All patients were breathing room air or supplemental oxygen by nasal cannula only.

To record lung sounds, a Littmann Cardiology III stethoscope chestpiece (3M, Maplewood, Minnesota, USA) was attached to an Eko Core electronic stethoscope attachment (Eko Devices, Berkeley, California, USA). This was attached to a 3-centimetre piece of stethoscope tubing with a lapel microphone (iGear Tech, Toronto, Ontario, Canada) inserted. Lung sounds were recorded with 
the Sony Audio Recorder Android app (Sony, Lund, Sweden). Recordings were produced with the Eko Core device in both the analogue mode, which is equivalent to a standard stethoscope, and the electronic mode. Auscultation was performed using the diaphragm side of the chestpiece, which has been shown to allow better characterisation of abnormal lung sounds than the bell. ${ }^{11}$ All analogue and electronic recordings were filtered by the same settings with the Adobe Audition CC Windows application (Adobe, San Jose, California, USA) to reduce artefact. Representative 5 -second clips were selected for each analogue and electronic recording.

Six fictional cases were written to represent ambulatory visits based on patients with the recorded pathology, as determined by brief review of the patient charts, imaging and discussion with providers. General pathology by case was as follows:

Case 1: acute exacerbation of COPD

Case 2: pancreatitis with coarse breath sounds at the left base

Case 3: pleural effusion

Case 4: congestive heart failure (CHF) exacerbation

Case 5: pneumonia

Case 6: pneumonia with wheezing

Cases were disseminated through an online application developed by the research team using Microsoft Visual Studio 2015 (Microsoft, Redmond, Washington, USA). Internal medicine residents were recruited by emails sent to administrators at programmes nationwide with the request that they be forwarded to their residents. Participants were asked to provide their residency programme email addresses to verify that they were internal medicine residents. Each participant was randomised to hear either analogue or electronic lung sounds when he or she registered on the website. The participant would only hear lung sounds in the same predetermined mode for all the cases but was not told which set of sounds he or she was hearing. In each case, the participant was asked to make a clinical decision by multiple choice selection based on the history and auscultation. Management plans included options such as ordering a chest X-ray to rule out pneumonia, treating for a COPD exacerbation, treating for CHF, evaluating for malignancy and conservative monitoring. In addition to the participant's selection, the number of times the participant listened to the lung sounds, as well as the time spent on each case, were tracked, as they may reflect efficiency and confidence in one's response.

\section{Data analysis}

The total numbers and proportions of correct answers were calculated for participants who completed all six cases (a.k.a. the module). Participants were permitted to stop at any time, and the analysis of each individual case included all participants who completed that case even if they did not complete the whole module. Case completions in which an answer was submitted without the participant having listened to any lung sounds were excluded. Differences between the analogue and electronic stethoscope were investigated in two ways: total scores (only the participants who completed all six cases) and by case (all participants who completed that case), using generalised linear mixed modelling (GLMM) with sandwich estimation assuming a binomial distribution (0-6) and (0/1), respectively. The time spent on each case and the numbers of times the participants listened to the recordings were compared for the two groups using GLMM assuming a negative binomial distribution. Because neither the numbers of times the recordings were played, nor time spent on the cases were distributed normally, Poisson modelling was used. However, evidence of overdispersion was found for the time spent on the cases, given an overdispersion parameter value of 21.5. Therefore, modelling using the negative binomial model was used for amount of time spent on the cases. Data were stored initially in an XML file, which was converted to a Microsoft Excel (Microsoft) spreadsheet when data collection was complete. Data analysis was performed with SAS Software V.9.4 (SAS) with the Generalized Linear Mixed Modeling (GLIMMIX) procedure.

\section{RESULTS}

About 61 internal medicine residents representing 23 programmes registered and completed at least one case, and 41 residents completed all six cases. The remaining 20 participants completed different numbers of cases but did not finish the whole module, which is why there were differences in sample size for the individual cases. For participants who completed all six questions, there were no significant differences in overall scores between participants who heard analogue (3.14 \pm 0.10 out of 6$)$ and electronic $(3.20 \pm 0.10$ out of 6) lung sounds $(p=0.74)$. Performance by case is shown in table 1 . Time spent listening and numbers of times the recordings were played by case are shown in tables 2 and 3, respectively. There were no significant differences in the numbers of correct answers for the two modalities for any of the six cases (overall $\mathrm{p}=0.78$ ). There were no significant differences in time spent on the cases or numbers of times the recordings were played for any of the six cases as well $(p=0.67$ and $p=0.85$, respectively).

\section{DISCUSSION}

In this simulated study of pulmonary auscultation by internal medicine residents, there was no observed overall improvement in accuracy when enhanced with an electronic stethoscope, though a larger sample size may have been needed to detect a difference if one exists. We also measured the amount

\begin{tabular}{|c|c|c|c|c|c|c|}
\hline \multirow[b]{2}{*}{ Question } & \multicolumn{2}{|l|}{ Analogue } & \multicolumn{2}{|c|}{ Electronic } & \multicolumn{2}{|l|}{ Difference } \\
\hline & $\begin{array}{l}\text { Number } \\
\text { correct }\end{array}$ & $\begin{array}{l}\text { Proportion } \\
\text { correct }\end{array}$ & $\begin{array}{l}\text { Number } \\
\text { correct }\end{array}$ & $\begin{array}{l}\text { Proportion } \\
\text { correct }\end{array}$ & OR $(95 \% \mathrm{Cl})$ & $P$ values \\
\hline 1 & $19 / 33$ & 0.58 & $21 / 28$ & 0.75 & 0.45 (0.15 to 1.37$)$ & 0.16 \\
\hline 2 & $25 / 30$ & 0.83 & $20 / 25$ & 0.80 & 1.25 (0.32 to 4.97$)$ & 0.75 \\
\hline 3 & $11 / 23$ & 0.48 & $16 / 23$ & 0.70 & 0.40 (0.12 to 1.35$)$ & 0.14 \\
\hline 4 & $15 / 23$ & 0.65 & $15 / 21$ & 0.71 & 0.75 (0.21 to 2.71$)$ & 0.66 \\
\hline 5 & $4 / 21$ & 0.19 & $6 / 21$ & 0.29 & 0.59 (0.14 to 2.51$)$ & 0.47 \\
\hline 6 & $8 / 21$ & 0.38 & $2 / 20$ & 0.10 & 5.54 (1.00 to 30.79$)$ & 0.05 \\
\hline
\end{tabular}


Table 2 Time spent listening

\begin{tabular}{|c|c|c|c|c|c|c|c|}
\hline \multirow[b]{2}{*}{ Question } & \multicolumn{2}{|c|}{ Analogue } & \multicolumn{2}{|c|}{ Electronic } & \multicolumn{3}{|c|}{ Comparison } \\
\hline & $\mathrm{N}$ & $\begin{array}{l}\text { Mean duration } \\
\text { (s) }\end{array}$ & $\mathrm{N}$ & $\begin{array}{l}\text { Mean duration } \\
\text { (s) }\end{array}$ & Ratio & $95 \% \mathrm{Cl}$ of ratio & $P$ values \\
\hline 1 & 33 & 86 & 28 & 104.1 & 0.83 & (0.63 to 1.08 ) & 0.16 \\
\hline 2 & 30 & 108.7 & 25 & 112.8 & 0.96 & (0.62 to 1.50$)$ & 0.87 \\
\hline 3 & 23 & 119.4 & 23 & 109.4 & 1.09 & (0.82 to 1.45 ) & 0.54 \\
\hline 4 & 23 & 99.1 & 21 & 97.3 & 1.02 & (0.74 to 1.40$)$ & 0.91 \\
\hline 5 & 21 & 102.5 & 21 & 120.4 & 0.85 & (0.55 to 1.32 ) & 0.47 \\
\hline 6 & 21 & 134.2 & 20 & 134.7 & 1.00 & (0.60 to 1.66$)$ & 0.99 \\
\hline
\end{tabular}

of time that the residents spent on each case and the numbers of times that they listened to the recordings to evaluate the hypothesis that amplification of lung sounds would make auscultation easier and allow for more rapid interpretation. However, we did not detect a difference between the analogue and electronic groups either in time spent listening or numbers of times the recordings were played.

Previous studies of pulmonary auscultation with electronic stethoscopes have demonstrated some objective and subjective benefits of this technology. The electronic stethoscope has been found to be useful in the noisy environment of military flight medicine, where physicians contend with jet engines to listen to lung sounds. When using an electronic stethoscope aboard a Boeing C135 military transport airplane, physicians rated it better for hearing heart and lung sounds. ${ }^{12}$ A study by the same group using a "Sim-Man" mannequin in an environment simulating that aboard a C135 demonstrated better performance on pulmonary auscultation by a sample of four intensivists and a nurse anaesthetist when using an electronic stethoscope. ${ }^{13}$ In a blinded study of pulmonary auscultation by anesthesiologists in the operating room, these physicians rated the quality of auscultation with the Littmann 3200 electronic stethoscope more highly than the analogue Cardiology III model. ${ }^{14}$ Notably, these studies were conducted in very different environments from ours, and outcomes for some of these studies were directed more towards personal preference than clinical decision making and outcomes.

The electronic stethoscope has also been shown to be beneficial in other studies of exclusively cardiac auscultation, such as in a Massachusetts General Hospital study in which a group of clinicians demonstrated improvement in identification of heart murmurs with an electronic stethoscope. ${ }^{15}$ In another study, medical students who received short-term training with an electronic stethoscope showed greater improvement from pretest to post-test in cardiac auscultation. ${ }^{16}$ In our study, however, we chose to study pulmonary rather than cardiac auscultation.
While our study did not demonstrate a benefit to amplification of lung sounds, electronic stethoscopes offer other capabilities, including computerised lung sound analysis (CLSA). ${ }^{1718}$ CLSA has been shown to improve performance and reliability, but a major drawback is that it is more difficult and time intensive to incorporate at the bedside than traditional auscultation. ${ }^{19}$ Furthermore, forgoing human auscultation altogether would reject a tradition that many clinicians and patients value. The development of devices that seamlessly incorporate CLSA with traditional auscultation at the bedside, perhaps using a smartwatch app or a visual display integrated into the device, would make the widespread use of this technology more feasible.

This study had several limitations. First, the clinical cases were designed to represent patient encounters, but this online simulation is not equivalent in-person pulmonary auscultation. Recordings were limited to one model of electronic stethoscope, and unlike in-person auscultation, participants could only listen to lung sounds from the six sites recorded for each patient and could not adjust their positioning. Additionally, our determination of a correct answer, although guided by clinical history and imaging as well as auscultation, is subjective. It is also possible that with a larger sample size we could have detected differences in performance that were not shown in this study (type II error). There was no type I error in this study, as none of the analyses reached statistical significance. Finally, this study was limited to internal medicine residents and may not represent clinicians in all specialties and stages of training.

\section{CONCLUSION}

In this study, amplification of lung sounds with an electronic stethoscope was not shown to affect overall performance on lung auscultation, nor was it shown to affect listener efficiency, which is likely related to confidence in one's interpretation. Further study is needed to confirm

Table 3 Numbers of times recordings were played

\begin{tabular}{|c|c|c|c|c|c|c|c|}
\hline \multirow[b]{2}{*}{ Question } & \multicolumn{2}{|c|}{ Analogue } & \multicolumn{2}{|c|}{ Electronic } & \multirow[b]{2}{*}{ Ratio } & \multirow[b]{2}{*}{$95 \% \mathrm{Cl}$ of ratio } & \multirow[b]{2}{*}{$P$ values } \\
\hline & $\mathrm{N}$ & Count (mean) & $\mathrm{N}$ & Count (mean) & & & \\
\hline 1 & 33 & 7.3 & 28 & 6.8 & 1.08 & (0.91 to 1.28 ) & 0.37 \\
\hline 2 & 30 & 7 & 25 & 7 & 1.01 & (0.82 to 1.24 ) & 0.96 \\
\hline 3 & 23 & 6.9 & 23 & 6.3 & 1.09 & (0.89 to 1.33 ) & 0.40 \\
\hline 4 & 23 & 5.1 & 21 & 5.8 & 0.88 & (0.71 to 1.08$)$ & 0.22 \\
\hline 5 & 21 & 5.1 & 21 & 5.3 & 0.96 & (0.77 to 1.20 ) & 0.75 \\
\hline 6 & 21 & 5.2 & 20 & 5.6 & 0.94 & (0.72 to 1.21 ) & 0.61 \\
\hline
\end{tabular}




\section{Main messages}

- The electronic stethoscope is an enticing innovation to keep auscultation relevant in the age of advanced imaging but its benefit is uncertain.

- In this study, the use of an electronic stethoscope for pulmonary auscultation was not shown to significantly affect clinical decision making, amount of repeat auscultation or time spent on clinical cases.

- Other capabilities of electronic stethoscopes such as computerised lung sound analysis (CLSA) require further study to determine how they may be used to augment auscultation at the bedside.

\section{Current research questions}

- Are there certain instances or types of pathology where electronic amplification is beneficial even if it does not confer an overall benefit?

- Does the electronic stethoscope have more benefit for cardiac than pulmonary auscultation?

- Is there an educational role for electronic stethoscopes for medical students and junior physicians?

- Is there a role for CLSA in routine bedside care?

\section{What is already known on the subject}

- Electronic stethoscopes have been shown to have subjective and objective benefits for cardiac and pulmonary auscultation in environments as diverse as hospital wards, operating rooms, medical schools and military transport airplanes.

- Advanced processing technologies such as computerised lung sound analysis (CLSA) improve the reliability of auscultation.

our results with more statistical power, identify specific types of pathology where the added sensitivity of an electronic stethoscope might be beneficial, and to evaluate the clinical utility of advanced processing technologies at the bedside.

Acknowledgements We appreciate the assistance of Jason Machan, PhD and Alison Chambers PhD with study design, and of Grayson Baird, PhD with data analysis.
Contributors ERG, JMA, DT: participated in the design of this study and writing of the manuscript. ERG: conducted data collection and analysis.

Funding The authors have not declared a specific grant for this research from any funding agency in the public, commercial or not-for-profit sectors.

Competing interests None declared.

Patient consent Not required.

Ethics approval This study was approved by the institutional review board (IRB) for Lifespan/Rhode Island Hospital.

Provenance and peer review Not commissioned; externally peer reviewed.

Data sharing statement Data and material are available upon request.

\section{REFERENCES}

1 David L, Dumitrascu DL. The bicentennial of the stethoscope: a reappraisal. Clujul Med 2017;90:361-3.

2 Rice T. 'The hallmark of a doctor': the stethoscope and the making of medical identity. Journal of Material Culture 2010;15:287-301.

3 Ologunde R, Rabiu R. Stetho-hammer: a not-so-novel aid in the neurologic examination. Surgery 2014;156:1286-7.

4 Welsby PD. Stethoscope is a hearing aid. BMJ 2010;341.c5985.

5 Rufai SR, Hamami H, Devoto L. Using the "Stethoscope Test" to improve preoperative diagnosis for appendicitis in children. J Visc Surg 2017;154:307-8.

6 Torino C, Gargani L, Sicari R, et al. The agreement between auscultation and lung ultrasound in hemodialysis patients: the LUST study. Clin J Am Soc Nephrol 2016:11:2005-11.

7 Peacock WF, Harrison A, Moffa D. Clinical and economic benefits of using AUDICOR S3 detection for diagnosis and treatment of acute decompensated heart failure. Congest Heart Fail 2006;12-32-6.

8 Butterworth JS, Poindexter CA. A multiple channel electronic stethoscope for teaching and demonstration. Am Heart J 1948:36:112-4.

9 Leng S, Tan RS, Chai KTC, et al. The electronic stethoscope. Biomed Eng Online 2015;14:1-37.

10 Wong V. Mobile device-based stethoscope system. US 20150201272, United States: Patent and Trademark Office, 2015

11 Welsby PD, Earis JE. Some high pitched thoughts on chest examination. Postgrad Med J 2001:77:617-20

12 Tourtier JP, Libert N, Clapson P, et al. Auscultation in flight: comparison of conventional and electronic stethoscopes. Air Med J 2011;30:158-60.

13 Tourtier JP, Libert N, Clapson P, et al. A simulator-based study of in-flight auscultation. Simul Healthc 2014;9:81-4.

14 Hoffmann C, Falzone E, Verret C, et al. Brief report: pulmonary auscultation in the operating room: a prospective randomized blinded trial comparing electronic and conventional stethoscopes. Anesth Analg 2013:117:646-8.

15 Kelmenson DA, Heath JK, Ball SA, et al. Prototype electronic stethoscope vs. conventional stethoscope for auscultation of heart sounds. J Med Eng Technol 2014;38:307-10

16 Mesquita CT, Reis JC, Simões $L S$, et al. Digital stethoscope as an innovative tool on the teaching of auscultatory skills. Arq Bras Cardiol 2013;100:187-9.

17 Gurung A, Scrafford CG, Tielsch JM, et al. Computerized lung sound analysis as diagnostic aid for the detection of abnormal lung sounds: a systematic review and meta-analysis. Respir Med 2011;105:1396-403.

18 Kevat AC, Kalirajah A, Roseby R. Digital stethoscopes compared to standard auscultation for detecting abnormal paediatric breath sounds. Eur J Pediatr 2017:176:989-92.

19 Murphy RL. In defense of the stethoscope. Respir Care 2008;53:355-69. 